\title{
Accurate electronics calibration for particle backscattering spectrometry
}

\author{
J.L. Colaux \& C. Jeynes \\ University of Surrey Ion Beam Centre, Guildford GU2 7XH, England
}

\begin{abstract}
Rutherford backscattering spectrometry (RBS) is a non-destructive thin film analytical technique of the highest absolute accuracy which, when used for elemental depth profiling, depends at first order on the gain of the pulse-height spectrometry system. We show here for the first time how this gain can be reliably and robustly determined at about $0.1 \%$.
\end{abstract}

Keywords:

Pulse-height defect, CRM, quantity of material, RBS, ISO 17025

A version of this paper was accepted by Analytical Methods $29^{\text {th }}$ January 2015, and subsequently published as Anal. Meth., 7 (2015) 3096-3104. 


\section{Introduction: the value of high accuracy RBS}

High accuracy Rutherford backscattering spectrometry (RBS) is a powerful analytical method for metrology purposes. We have recently demonstrated that high accuracy RBS can be used for validating the accuracy of our implanted fluence measurement both by charge collection and also by sheet resistance measurements ${ }^{1,2}$. This is an important result showing that RBS and quantitative implantation are both suitable for certifying new ion implanted or other standards for the quantification of other analytical techniques such as secondary ion mass spectrometry (SIMS) or Xray fluorescence (XRF). High accuracy RBS will also prove very valuable in Total-IBA (the synergistic use of multiple IBA techniques, such as RBS and PIXE) where the other techniques can inherit the accuracy of RBS ${ }^{3}$.

Equations $1 \& 2$ describe RBS and have been explained in great detail previously ${ }^{4}$. They have been simplified without any loss of generality, and their simplicity underlines why RBS is expected to be a high accuracy technique.

$$
\begin{array}{ll}
A=Q N \sigma \Omega & \text { Eq.1 } \\
Y=Q \sigma \Omega \Delta /[\varepsilon] & \text { Eq.2 }
\end{array}
$$

Figure 1 shows a pair of RBS spectra from an arsenic-implanted silicon sample. Considering the question of how many As atoms there are in the sample, Eq.1 simply says that the number of counts $A$ in the As signal is given by the product of: the number of He particles $Q$ in the probing beam, the number per unit area $N$ of As atoms, the probability $\sigma$ of the He scattering from the As (expressed as an area), and the probability of detection $\Omega$ (expressed as a detector solid angle).

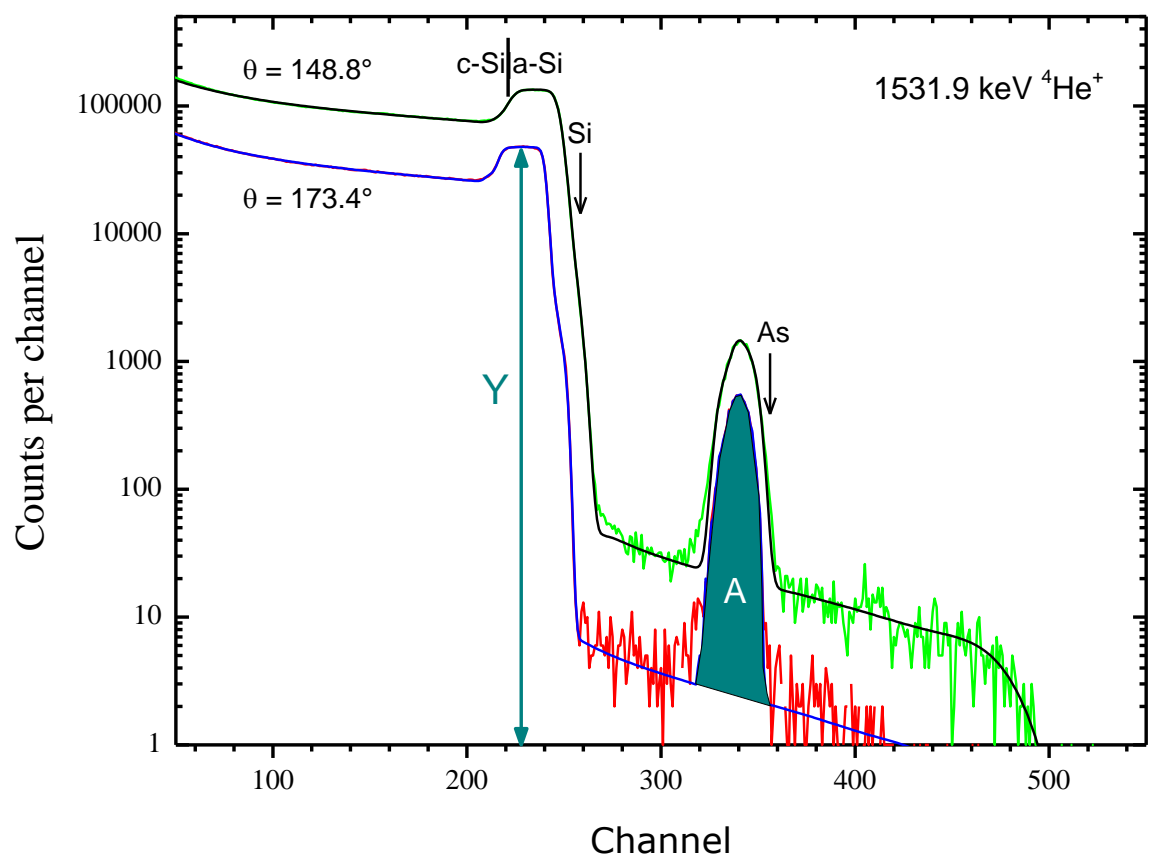

Figure 1: RBS (green \& red) pulse-height spectra of a $150 \mathrm{keV} 10^{15} \mathrm{As} / \mathrm{cm}^{2}$ implant, with fits (black \& blue). Spectra are collected in two detectors from 13 separate spots mapping the wafer: the two summed spectra are shown. The As and $S$ elemental edges and the pileup signal are marked for the upper spectrum (DetB; $\theta=149^{\circ}$ ). The beam is aligned with the single crystal substrate: the amorphous - crystalline interface is also marked (c-Si|a-Si). The DetA spectrum is similar. $A$ (Eq.1) and $Y$ (Eq.2) are marked for the lower (DetA; $\theta=173^{\circ}$ ) spectrum. 
Usually, a scattering cross-section function $(\sigma)$ is complicated and not known very well, but if the incident beam energy is low enough so that the two nuclei involved in the scattering event do not overlap, then $\sigma$ is given by the analytical Rutherford formula which simply assumes Coulomb repulsion of point charges. Spectra shown in Figure $\mathbf{1}$ are purely Rutherford. Determining a fluence $N$ from a spectral area $A$ therefore requires knowledge of the charge $\times$ solid-angle product $(Q \Omega)$, but as we have discussed at length elsewhere ${ }^{2}$ the charge $Q$ is not easy to measure accurately, and accurate measurements of the solid-angle $\Omega$ are also notoriously difficult. Therefore, referring to Equation 2, we can obtain $Q \Omega$ from the amorphous silicon yield $Y$ (counts/channel), the gain $\Delta$ (keV/channel) of the spectroscopic electronics and the energy loss factor $[\varepsilon]$, the latter being a physical constant corresponding to the integrated inelastic energy loss of the $\mathrm{He}^{+}$ion in the sample, both on the in-path to the scattering event and the out-path towards the detector (in eV/TFU, where TFU $\equiv$ "thin film unit" $\equiv 10^{15}$ atoms $/ \mathrm{cm}^{2}$ ). In this case $[\varepsilon]$ has been measured at $0.8 \%$ uncertainty ${ }^{1}$ relative to an Sb-implanted certified reference material (CRM) ${ }^{5}$. But the channel width $\Delta(\mathrm{keV} / \mathrm{channel})$ is the gain of the spectroscopic electronics, and must be measured for each detection channel every day, and every time the gain is changed. The present work shows how to determine this electronic gain very accurately.

Figure 1 shows spectra taken from our programme for the quality assurance (QA) of our quantitative implantation, which is discussed in detail elsewhere ${ }^{2}$. The statistics in these spectra are very good since the signals of interest are rather small and we want a $1 \%$ measurement accuracy. It is interesting that the isotopic abundance of silicon is very clearly modelled in these data, and also that the pulse pileup is equally well modelled. What is critical is that there are two detectors in the scattering chamber: this means that we immediately have a double-check on the gain determination since the two independent detector channels have to give the same As fluence. And since the channels are independent, the average result has $\sqrt{2}$ of the uncertainty of each. In terms of the Guide to the Expression of Uncertainty in Measurement (GUM) ${ }^{6}$ we can give a Type $A$ estimate of uncertainty instead of a (rather uncertain) Type $B$ one; thus two detectors are infinitely better than one, but four detectors are only $\sqrt{2}$ as good as two: there are rapidly diminishing returns!

The present work is one of a set of five papers that unequivocally establishes high accuracy RBS as a definitive method for the traceable and non-destructive determination of quantity of material in thin films at a global accuracy of $1 \%$. In 2012, an interlaboratory study of Jeynes, Barradas \& Szilágyi ${ }^{4}$ demonstrated reproducibility at about $1 \%$, also describing RBS in detail including second (and higher) order effects and claiming $\sim 0.1 \%$ (Type B) for the gain uncertainty (without any details). In 2014 the longitudinal study of Colaux \& Jeynes ${ }^{1}$ demonstrated reproducibility at about $0.9 \%$, also establishing the uncertainty budget in considerable detail but also giving no details of how to achieve the claimed accuracy ( $0.2 \%$, Type A) for the gain calibration. A companion paper to the present one ${ }^{7}$ concentrates on a straightforward method, using the ${ }^{16} \mathrm{O}(\alpha, \alpha)^{16} \mathrm{O}$ resonance at $3038 \mathrm{keV}$, for establishing the beam energy at $0.07 \%$. In the present work we explicitly justify our Type A estimate of about $0.1 \%$ for the uncertainty of the gain determination, also showing why it is easy to underestimate it by consideration of the covariance factors. The single-measurement 
reproducibility (of $0.99 \%$ ) for this high accuracy RBS method as well as the accuracy of about $1 \%$ for quantitative implantation at Surrey are treated fully quantitatively in the last of this 5-paper set ${ }^{2}$.

Previous claims of high accuracy determination of electronic gain include Gurbich \& Jeynes ${ }^{8}$, who also estimate $0.1 \%$ accuracy and discuss the issue. Their claim is credible since a proton beam was being used (with a much smaller pulse-height defect, or PHD), together with a very wide range of energies (as here). The previous authors they cite did not justify their claims - except for Munnik et $a l^{9}$, who also treat a wide range of energies, and who also correctly and explicitly treat the PHD. Munnik et al estimated a standard error on their gain of $0.16 \%$.

\section{Experimental details}

RBS measurements used the 6-axis goniometer of the $2 \mathrm{MV}$ Tandem accelerator of the University of Surrey Ion Beam Centre ${ }^{10}$ which allows air-lock handling of $100 \mathrm{~mm}$ wafers without breaking vacuum. Two standard semiconductor diode detectors were used at backscattering angles of $173.4^{\circ}$ (DetA) and $148.8^{\circ}$ (DetB), measured with an accuracy of $0.2^{\circ}$ using the goniometer with an in-line laser. The solid angles of detection were 0.9 and $2.1 \mathrm{msr}$ for DetA and DetB, respectively.

The beam energy is controlled using feedback from the generating voltmeter (GVM) monitoring the tandem terminal voltage. The GVM calibration factor (i.e. relationship between the nominal and actual terminal voltage) is determined with an absolute accuracy of $0.07 \%$ as described elsewhere ${ }^{7}$.

Standard analogue electronics were used for pulse-height amplification and measurement with successive-approximation ( $6 \mu$ s conversion time) ADCs. The shaping amplifiers have a shaping time of about 500 ns, and implement a pulse-pileup inspection circuit with a time resolution also of about 500 ns: the ADCs were gated to reject detected pileup events.

The ADC electronic zero ("offset") was measured directly using an electronic pulser: that is, electronic pulses of various heights (measured with a storage oscilloscope) are recorded by the pulse-height spectrometry system. The offset (in channels) is determined from a linear regression of the pulse-height (in volts) and the pulse position (in channel numbers). The offset in keV follows from knowing the electronic gain of the pulse-height spectrometry system (keV/ch).

The electronic gains obtained by our calibration method are validated against the Sb-implant certified reference material (CRM). The certified ion fluence is $(48.1 \pm 0.6) \times 10^{15} \mathrm{Sb} / \mathrm{cm}^{2}$, where the stated expanded combined uncertainty has coverage factor $k=2$. The CRM is a $15 \mathrm{~mm}$ square piece of IRMM-ERM-EG001/BAM-L001 ${ }^{5}$, subsequently amorphised at Surrey to a depth of about $630 \mathrm{~nm}$ with an "Epifab" implant ${ }^{11}$ : that is, a $5 \times 10^{15}{ }^{28} \mathrm{Si} / \mathrm{cm}^{2}$ cold implant at $500 \mathrm{keV}$ on a liquid-nitrogen cooled stage. It is necessary to amorphise the CRM since we need the silicon yield $Y$ (Eq.2) to be unaffected by channelling effects: it has long been recognised that methods of "randomising" the beam direction into single crystals to avoid RBS channelling effects are only effective at accuracies of $4 \%$ or so (there are no "random" directions in single crystals) ${ }^{12}$, not good enough for the present purposes. 
The RBS spectra were fitted using the DataFurnace code ${ }^{13}$ with executable versions NDFv9.6a and WiNDFv9.3.76 ${ }^{14}$. This code implements Andersen screening ${ }^{15}$, SRIM-2003 stopping powers ${ }^{16}$ (note that the latest SRIM ${ }^{17}$ is not materially different, and we have demonstrated previously that SRIM-2003 is correct at $0.8 \%$ for $\mathrm{Si}^{1}$ ), Molodtsov \& Gurbich pileup correction ${ }^{18}$, and also the pulseheight defect (PHD) correction of Pascual-Izarra \& Barradas ${ }^{19}$ which uses Lennard's calculation of the non-ionising energy loss ${ }^{20}$. The channelled substrate signals were fitted using an ad-hoc cubic polynomial correction to the scattering cross-section of $\mathrm{Si}$, discussed in detail by Barradas et $a^{21}$. A parameterisation of the measurements of Pascual-Izarra et $a l^{22}$, which have been confirmed by Lennard et $a l^{23}$ (who gives his results in terms of the Si stopping powers of Konac et al ${ }^{24}$ ), were used for the $\mathrm{SiO}_{2}$ stopping power.

In this work spectral fitting is accomplished by minimising the standard chi-squared $\left(\chi^{2}\right)$ function (using the usual definition of $\chi^{2}$ : the sum over all channels of the squared deviation of the simulated from the collected spectra). Any convenient function can be minimised, but $\chi^{2}$ is wellbehaved where the simulation is close to the data. DataFurnace can use other functions for minimisation, the "robustified $\chi^{2 "}$ is discussed in the companion work ${ }^{5}$.

\section{Calibrating the pulse-height spectrometry system}

The electronic gain $(\Delta)$ of the acquisition system is usually derived from a spectrum obtained from a calibration sample such as shown in Figure 2. This is a multilayer calibration sample discussed at length previously ${ }^{4,25}$ and consisting of a thin metallic bilayer on a silica-coated silicon substrate. Thus, there are four elemental signals ( $\mathrm{Au}, \mathrm{Ni}, \mathrm{Si} \& \mathrm{O})$, and with a detector at a given scattering angle we will detect particles backscattered from the "surface" of the sample whose energy can be calculated precisely from the incident beam energy and a simple application of the kinematics of the scattering event. Of course, the signals in this case from $\mathrm{Si}$ and $\mathrm{O}$ are not exactly surface signals: they are noticeably displaced to lower energies because of the energy loss through the metal layers, but the energy loss in the metals is known well enough for this not to compromise the available calibration precision.

With reasonable care, treating the spectra as energy spectra allows one to routinely achieve accuracies around $2 \%$. But this is not good enough for the technique to be taken seriously as a "definitive method" ${ }^{26}$, nor is it good enough for straightforward QA applications, such as the important case of implanter fluence qualification treated in the companion work ${ }^{2}$. Lennard pointed out forcefully some time ago ${ }^{23}$ that the traditional RBS spectra obtained using semiconductor detectors as shown in Figures $1 \& 2$ are pulse-height spectra, not energy ones. The pulse-height response of such detectors is proportional only to that part of the particle energy that is converted to electron-hole pairs in the active region of the detector, but some of the particle energy is also lost both at the entrance window and into the nuclear displacements which are not converted to electron-hole pairs. This is known as the pulse-height defect (PHD) which, as Figure $\mathbf{3}$ shows, varies quite strongly with energy ${ }^{20,27}$ and is not quite the same as a simple offset strictly independent of energy. The PHD must be taken into account for properly interpreting the RBS spectra. 


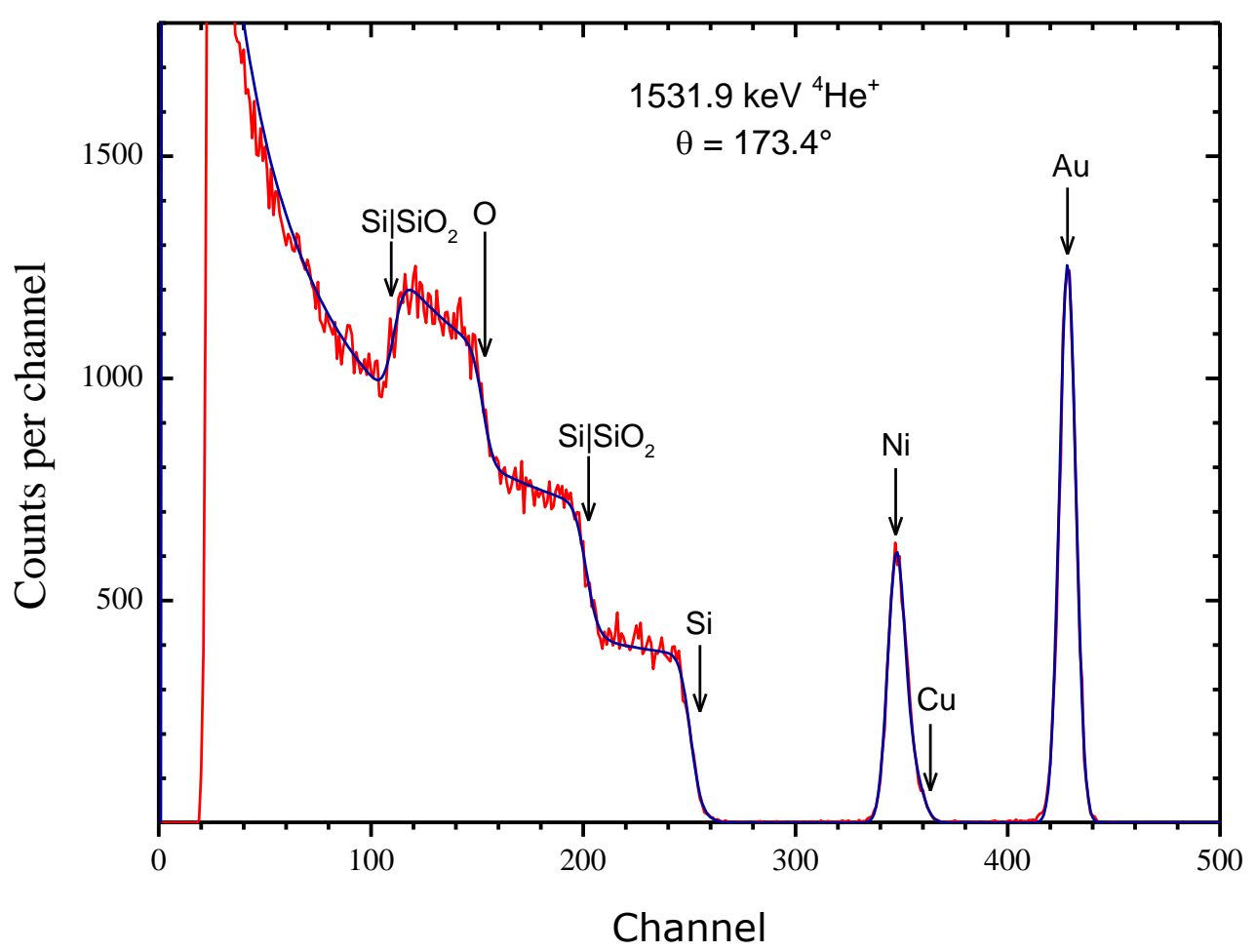

Figure 2: RBS pulse-height spectrum (red) from the standard calibration sample, with fit (blue: see text). This sample has a nominal structure $(10,20,2200)$ TFU of $\left(\mathrm{Au}, \mathrm{Ni}, \mathrm{SiO}_{2}\right.$ : TFU $\equiv$ "thin film unit" $\left.\equiv 10^{15} \mathrm{atoms} / \mathrm{cm}^{2}\right)$. This is equivalent to $(1.7,2.2,333) \mathrm{nm}$ at full bulk density. The fit is shown in blue. The collected charge is about $10 \mu \mathrm{C}$. The Ni actually has about $10 \% \mathrm{Cu}$, as confirmed by PIXE. There is channelling on the Si substrate fitted by an ad hoc cubic polynomial: higher order terms are needed at low energies because of multiple scattering and other effects. Elemental edges at (428, 347, $251,153)$ channels are shown, together with the interface positions in the Si and O signals.

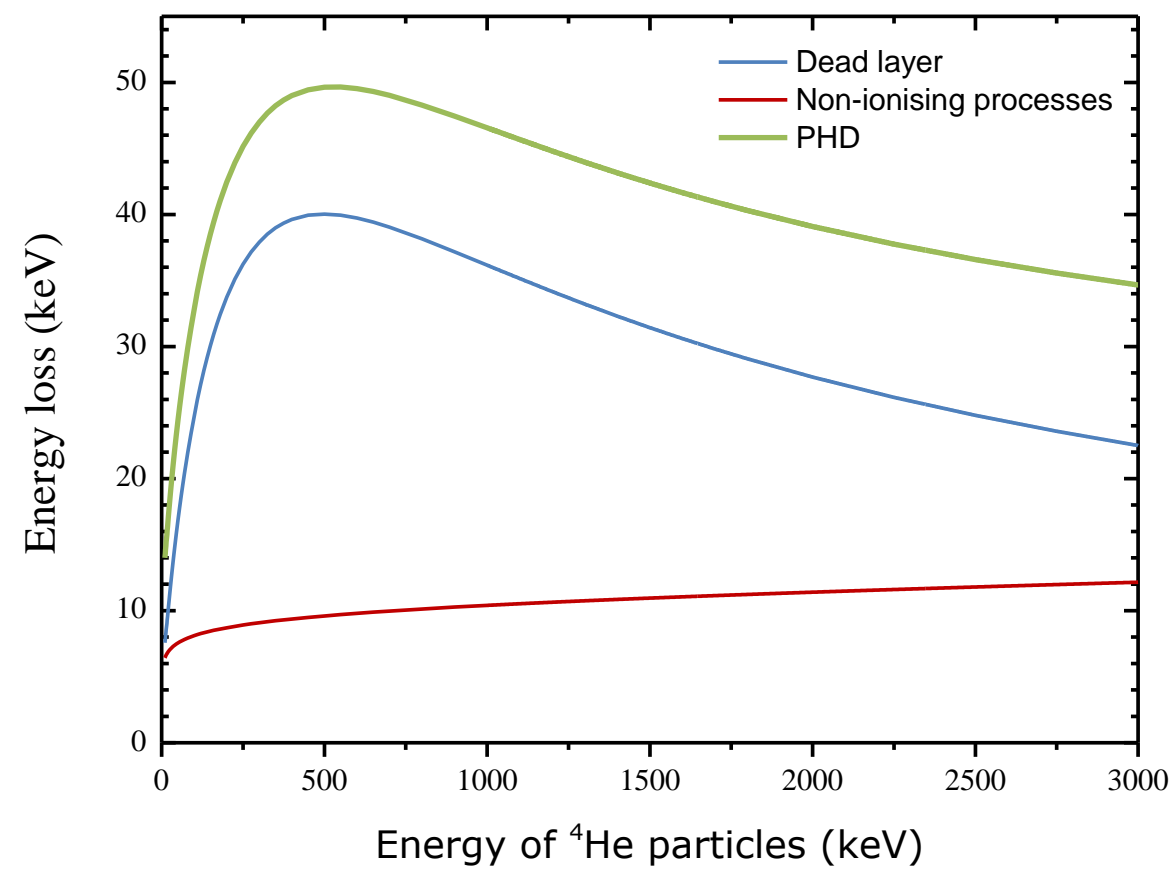

Figure 3: PHD energy loss function for ${ }^{4} \mathrm{He}$ particles. The PHD (green curve) is the sum of the energy losses due to the dead layer (blue curve, calculated for $590 \mathrm{TFU}$ of Si) and the non-ionising (nuclear) processes (red curve, calculated with Lennard's model ${ }^{20}$ ). 
The non-linearity of the PHD has a dramatic effect on RBS spectra that cannot be accurately fitted for all peaks and edges without properly taking it into account (see Fig.1 in Jeynes et $a l^{4}$ and Fig.4 in Pascual-Izarra \& Barradas ${ }^{19}$ ). On the other hand, Figure 3 shows that the non-linearity of the PHD is mainly due to the energy loss in the detector dead layer. In this work, we propose to use the silicon $\left(\mathrm{Si} \& \mathrm{Si} \mid \mathrm{SiO}_{2}\right)$ and oxygen $\left(\mathrm{O} \& \mathrm{Si} \mid \mathrm{SiO}_{2}\right)$ interface signals (see Figure 2$)$ for assessing the dead layer thickness used in DataFurnace to simulate the spectra. Obviously, using a single energy for determining the dead layer thickness is not quite good enough since the PHD function would only be probed on a rather small energy range (i.e. Si and $\mathrm{O}$ signals edges appear between 400 and $850 \mathrm{keV}$ in Figure 2). The use of a dataset acquired for a wide energy range is therefore required for properly deriving the detector dead layer thicknesses. In this work we used 18 pairs of spectra from two detectors: 9 pairs collected around the $3038 \mathrm{keV}{ }^{16} \mathrm{O}(\alpha, \alpha)^{16} \mathrm{O}$ resonance (this sub-set is also used for the GVM calibration procedure ${ }^{7}$ ) and 9 pairs collected at energies ranging from typically 1400 to $1700 \mathrm{keV}$ (see Table 1). From these 18 pairs of spectra, we want to extract the dead layer of each detector and the electronic gain of each detection channel. But these parameters are strongly correlated ${ }^{28}$ and an iterative procedure (see flow diagram in Figure 4) must be used to converge on the optimum values.

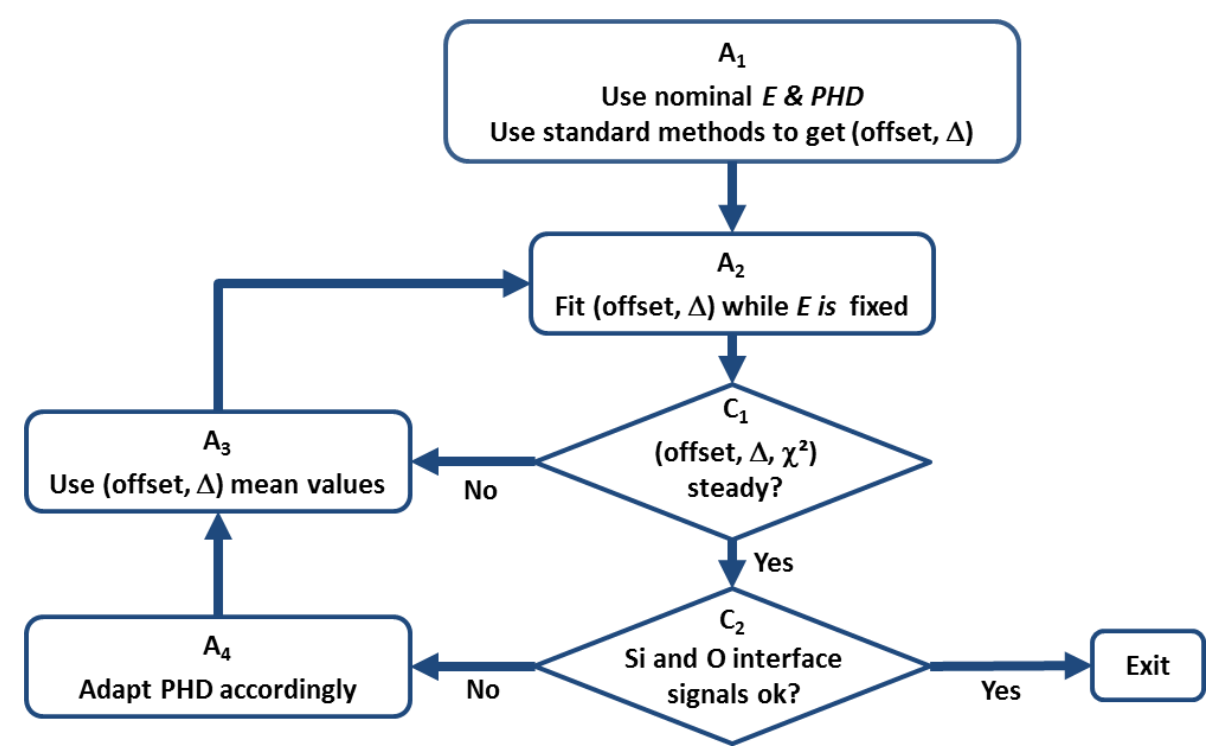

Figure 4: Flow diagram of the electronic gain calibration procedure. $E$ is the incident beam energy; offset and $\Delta$ are the electronic offset and gain of the pulse-height spectrometry system; and $\chi^{2}$ is the chi-squared calculated for each spectral fit. The output of this procedure is the gain, offset and pulse-height defect (PHD) for each detector.

$A_{1}$ The incident beam energies $(E)$ are calculated using the nominal terminal voltage and the GVM calibration factor of the accelerator ${ }^{7}$. The nominal PHD value may come from the datasheet of the detector; from previous work or from direct measurement using a triple-alpha source ${ }^{20}$. When no information is available, the PHD value is roughly determined by fixing the electronic offset at the value directly measured with an electronic pulser. 
$A_{2}$ The whole dataset (18 pairs of spectra) is fitted using DataFurnace, that is, all spectra are automatically fitted one after the other where the incident energy and PHD are fixed to nominal values and the electronic gain $( \pm 2 \%)$ and offset ( \pm 10 channels) are fitted to minimise the chi-squared $\left(\chi^{2}\right)$ function for each spectrum. Other parameters such as the collected charge $( \pm 20 \%)$, the detector resolution $( \pm 10 \mathrm{keV})$ and the sample depth profile are also allowed to vary during the $\chi^{2}$ minimisation. This is because, although there is a true value for all these (which ought to be known), any error in selected (non-varying) values will bias the results quite strongly. In fact, the data determine all of these parameters independently.

We should comment that the detector resolution is fitted because $\chi^{2}$ minimisation is extremely sensitive to the shape of edges and peaks, and if the instrumental function is not correct the positions of both edges and peaks can be heavily distorted. The purpose is to use these features of the spectra to determine the experimental parameters so that it is of the essence to avoid such distortion. Of course it is the position of the peaks and edges that fixes the electronic gain and offset. The precision with which these positions can be determined depends only on the size of the signals (and not on the nominal energy resolution), as was pointed out long ago in this context ${ }^{29}$. The manual methods used in Jeynes et al ${ }^{25}$ are equivalent to the automated $\chi^{2}$ fitting implemented by DataFurnace.

$C_{1}$ The results of the fitting procedure $\left(A_{2}\right)$ are exported to a spreadsheet where the mean and standard deviation (SD) of each fitted parameter are calculated and recorded for tracking their trend (shown in Figure 5) as a function of fitting iteration number. If the (offset, $\Delta, \chi^{2}$ ) mean values have not reached a steady state or if their SD is not satisfying, these mean values are used as inputs $\left(A_{3}\right)$ for performing the next fitting iteration $\left(A_{2}\right)$.

$\mathrm{C}_{2}$ When the criterion $\mathrm{C}_{1}$ is fulfilled, the analyst looks closely at the silicon ( $\mathrm{Si} \& \mathrm{Si} \mid \mathrm{SiO}_{2}$ ) and oxygen ( $\mathrm{O} \& \mathrm{Si} \mid \mathrm{SiO}_{2}$ ) interface signals (see Figure 2 ). If all of these edges are not properly fitted for each spectrum, the PHD value is accordingly adjusted $\left(A_{4}\right)$ for performing the next iteration $\left(A_{3}+A_{2}\right)$. Otherwise, the fitting procedure is completed.

It should be noted that both oxygen and silicon edges at the $\mathrm{Si} \mid \mathrm{SiO}_{2}$ interface are subject to possible errors existing in the $\mathrm{SiO}_{2}$ stopping power function. Since the exit path length is quite different for the two detectors, we separately fit the spectra recorded by DetA \& DetB, so that any error in the stopping power function can be artificially compensated by adapting the $\mathrm{SiO}_{2}$ thickness used for the simulation. The use of the correct stopping power function is also of importance for getting the correct electronic gain (see Discussion below). 


\section{Results}

Fitting the whole dataset (36 spectra: 2 detectors, 18 energies) with DataFurnace and exporting the results to a spreadsheet, one can obtain a table similar to Table 1 where the standard error at $99 \%$ of confidence (SE99) is given by:

$$
S E 99=\left(S D \times \sqrt{\frac{N-1}{\operatorname{Chiinv}\left(1-\frac{1-\alpha}{2} ; N-1\right)}}\right) / \sqrt{N-1} \quad \text { Eq.3 }
$$

where $\boldsymbol{S D}$ is the standard deviation of the $\boldsymbol{N}$ measurements, $\boldsymbol{\alpha}$ is the interval of confidence (i.e. 99\%) and Chiinv is the function that returns the inverse of the right-tailed probability of the chisquared distribution.

Logging the mean value of the fitted parameters (electronic gain, offset, PHD and $\chi^{2}$ ) after each iteration ( $A_{2}$ in Figure 4), one obtains the trend charts shown in Figure 5 and used for assessing the criterion $C_{1}$. In this example, steady state of all fitted parameters is reached fairly quickly ( 30 iterations) because of the prior knowledge available for the detectors: both PHD and electronic gain were previously derived (at lower accuracy) from the GVM calibration method ${ }^{7}$ using the subset of spectra acquired around $3 \mathrm{MeV}$.

At the end of this calibration procedure, the electronic gains are validated against any convenient ion-implanted standard sample. That is, if the electronic gains are correctly determined, both detectors ought to give the same (correct) measured fluence. We have used the Sb-implanted CRM for which the true (certified) value is known to be $(48.1 \pm 0.3) \times 10^{15} \mathrm{Sb} / \mathrm{cm}^{2}$ : the mean measured fluence ought to agree with this certified value. The results obtained over the last 18 months are summarised in Table 2. 
Submission to Analytical Methods, $16^{\text {th }}$ December 2014

\begin{tabular}{|c|c|c|c|c|c|c|}
\hline Detector & $\begin{array}{c}\text { Indicated terminal } \\
\text { voltage }(\mathrm{kV})\end{array}$ & $\begin{array}{l}\text { Detector resolution } \\
\text { FWMH (keV) }\end{array}$ & $\begin{array}{c}\text { Beam energy } \\
\text { (keV) }\end{array}$ & $\begin{array}{l}\text { Electronic gain } \\
\text { (keV/ch) }\end{array}$ & $\begin{array}{l}\text { Electronic offset } \\
(\mathrm{keV})\end{array}$ & $\chi^{2}$ \\
\hline \multirow{18}{*}{ 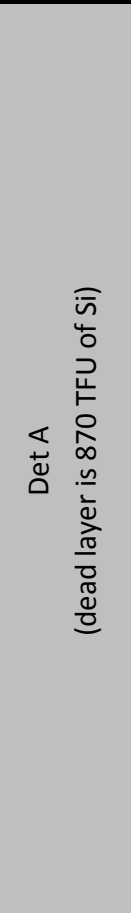 } & 1500 & 29.16 & 3044.3 & 3.1814 & -3.63 & 1.23 \\
\hline & 1503 & 28.87 & 3050.4 & 3.1808 & -3.50 & 1.17 \\
\hline & 1506 & 28.60 & 3056.4 & 3.1811 & -3.53 & 1.06 \\
\hline & 1509 & 28.72 & 3062.5 & 3.1824 & -3.58 & 0.91 \\
\hline & 1512 & 28.69 & 3068.5 & 3.1828 & -3.70 & 0.97 \\
\hline & 1515 & 28.55 & 3074.6 & 3.1829 & -3.69 & 0.98 \\
\hline & 1518 & 29.21 & 3080.6 & 3.1827 & -3.70 & 0.96 \\
\hline & 1521 & 28.51 & 3086.7 & 3.1838 & -3.61 & 0.94 \\
\hline & 1524 & 28.53 & 3092.7 & 3.1833 & -3.67 & 0.88 \\
\hline & 670 & 28.36 & 1370.6 & 3.1862 & -5.17 & 0.58 \\
\hline & 690 & 29.72 & 1410.9 & 3.1851 & -3.77 & 0.55 \\
\hline & 710 & 30.09 & 1451.3 & 3.1852 & -3.79 & 0.62 \\
\hline & 730 & 30.29 & 1491.6 & 3.1849 & -3.64 & 0.55 \\
\hline & 750 & 30.68 & 1531.9 & 3.1851 & -3.59 & 0.60 \\
\hline & 770 & 29.78 & 1572.3 & 3.1851 & -3.55 & 0.53 \\
\hline & 790 & 30.31 & 1612.6 & 3.1861 & -3.48 & 0.62 \\
\hline & 810 & 29.42 & 1652.9 & 3.1854 & -3.52 & 0.57 \\
\hline & 830 & 29.86 & 1693.3 & 3.1849 & -3.63 & 0.57 \\
\hline \multicolumn{2}{|c|}{ Average } & 29.3 & & 3.1838 & -3.7 & 0.8 \\
\hline \multicolumn{2}{|c|}{ SD } & $2.5 \%$ & & $0.05 \%$ & 0.4 & 0.2 \\
\hline \multicolumn{2}{|c|}{ SE99 (Eq.3) } & $4.4 \%$ & & $0.09 \%$ & & \\
\hline \multirow{18}{*}{ 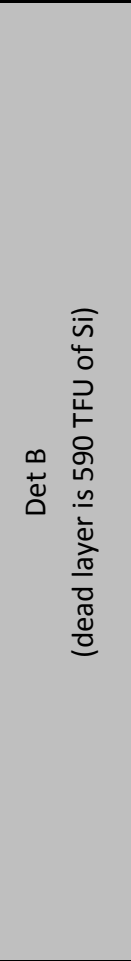 } & 1500 & 22.81 & 3044.3 & 2.9773 & 2.79 & 1.95 \\
\hline & 1503 & 22.58 & 3050.4 & 2.9778 & 2.75 & 1.93 \\
\hline & 1506 & 22.62 & 3056.4 & 2.9779 & 2.73 & 1.52 \\
\hline & 1509 & 22.46 & 3062.5 & 2.9782 & 2.77 & 1.49 \\
\hline & 1512 & 22.15 & 3068.5 & 2.9773 & 2.73 & 1.54 \\
\hline & 1515 & 22.16 & 3074.6 & 2.9779 & 2.70 & 1.51 \\
\hline & 1518 & 22.04 & 3080.6 & 2.9783 & 2.72 & 1.63 \\
\hline & 1521 & 21.75 & 3086.7 & 2.9782 & 2.74 & 1.56 \\
\hline & 1524 & 21.93 & 3092.7 & 2.9786 & 2.75 & 1.61 \\
\hline & 670 & 20.45 & 1370.6 & 2.9797 & 2.14 & 0.80 \\
\hline & 690 & 20.35 & 1410.9 & 2.9799 & 2.43 & 0.85 \\
\hline & 710 & 21.69 & 1451.3 & 2.9801 & 2.72 & 0.76 \\
\hline & 730 & 21.51 & 1491.6 & 2.9797 & 2.83 & 0.78 \\
\hline & 750 & 21.33 & 1531.9 & 2.9802 & 2.84 & 0.87 \\
\hline & 770 & 21.59 & 1572.3 & 2.9803 & 2.83 & 0.76 \\
\hline & 790 & 21.37 & 1612.6 & 2.9803 & 2.78 & 0.75 \\
\hline & 810 & 21.43 & 1652.9 & 2.9801 & 2.71 & 0.80 \\
\hline & 830 & 21.24 & 1693.3 & 2.9802 & 2.76 & 0.88 \\
\hline \multicolumn{2}{|c|}{ Average } & 21.7 & & 2.9790 & 2.7 & 1.2 \\
\hline \multicolumn{2}{|r|}{ SD } & $3.1 \%$ & & $0.04 \%$ & 0.2 & 0.4 \\
\hline \multicolumn{2}{|c|}{ SE99 (Eq.3) } & $5.4 \%$ & & $0.07 \%$ & & \\
\hline
\end{tabular}

Table 1: Final fitted results obtained in October 2014 following the energy calibration procedure described in Figure 4. 


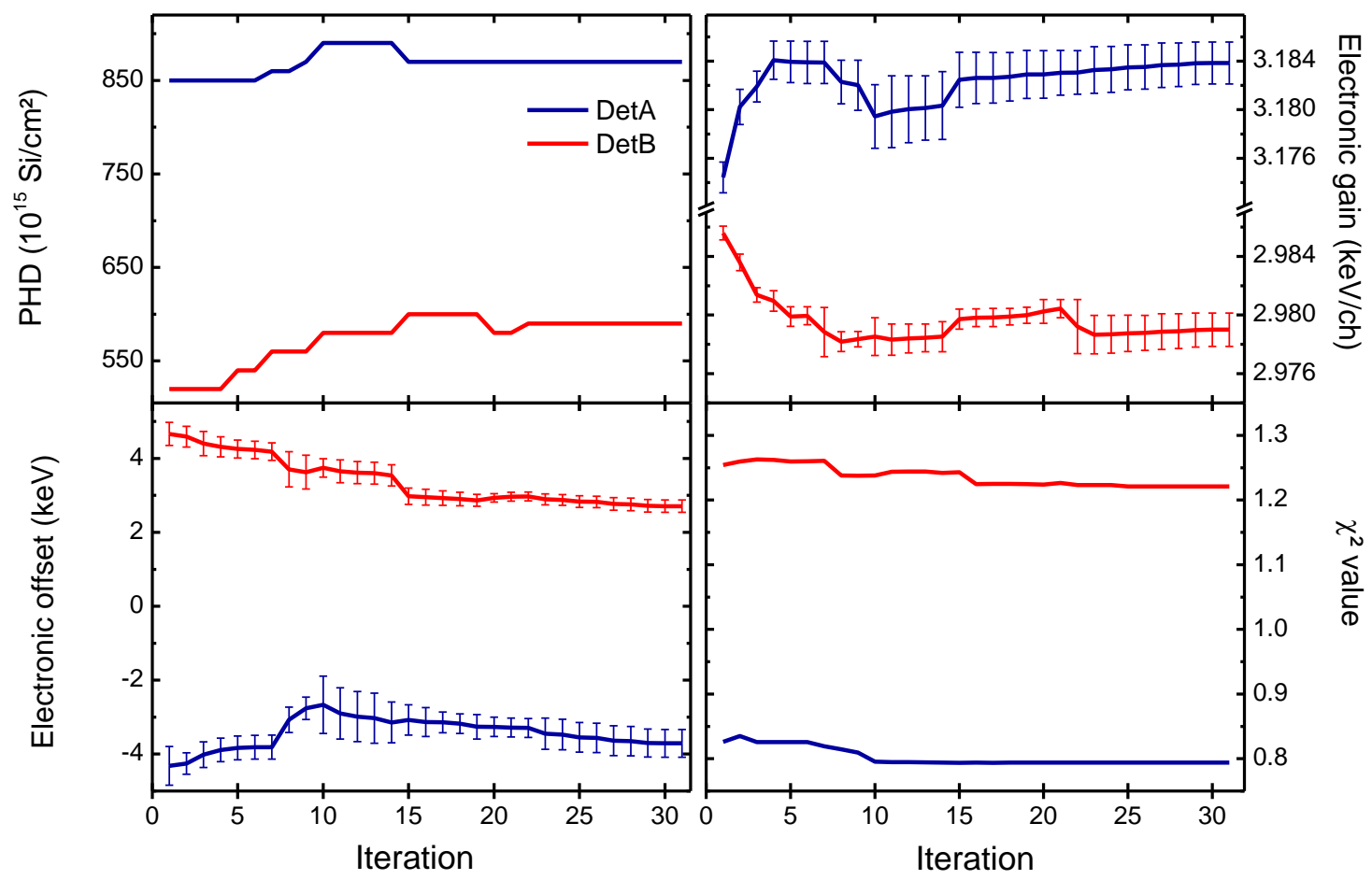

Figure 5: Evolution of the fitted parameters with iteration number. See Figure 4 for the gain calibration method. The fitted parameters are: electronic gain, offset, PHD and $\chi^{2}$. In the analysis shown, the electronic offset measured directly using an electronic pulser was found to be $(2.4 \pm 10.8) \mathrm{keV}$ for DetA and $(2.9 \pm 14.2) \mathrm{keV}$ for DetB. The standard error on these values is quite large due to the limited accuracy for the pulse-height measurement available with the oscilloscope.

\begin{tabular}{|c|c|c|c|c|c|c|c|c|c|c|}
\hline \multirow{2}{*}{ Date } & \multirow{2}{*}{$\begin{array}{c}\text { Certified } \\
\text { Sb-Fluence } \\
\text { (TFU) }\end{array}$} & \multirow{2}{*}{ 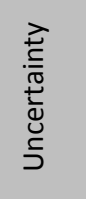 } & \multicolumn{3}{|c|}{$\begin{array}{l}\text { Measured Sb-Fluence } \\
\text { (TFU) }\end{array}$} & \multirow{2}{*}{ 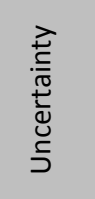 } & \multirow{2}{*}{$\begin{array}{c}\text { Ratio } \\
\text { DetA/DetB }\end{array}$} & \multirow{2}{*}{ 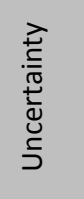 } & \multirow{2}{*}{$\begin{array}{c}\text { Ratio } \\
\text { Average/Certified }\end{array}$} & \multirow{2}{*}{ 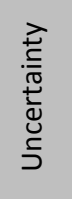 } \\
\hline & & & DetA & DetB & $\begin{array}{l}\text { Weighted } \\
\text { average }\end{array}$ & & & & & \\
\hline Jun-13 & 48.1 & $0.6 \%$ & 48.01 & 47.92 & 47.97 & $0.95 \%$ & 1.002 & $0.4 \%$ & 0.997 & $1.1 \%$ \\
\hline Jan-14 & 48.1 & $0.6 \%$ & 48.30 & 48.08 & 48.19 & $0.96 \%$ & 1.005 & $0.6 \%$ & 1.002 & $1.1 \%$ \\
\hline Jan-14 & 48.1 & $0.6 \%$ & 48.17 & 48.35 & 48.25 & $0.96 \%$ & 0.996 & $0.5 \%$ & 1.003 & $1.1 \%$ \\
\hline Apr-14 & 48.1 & $0.6 \%$ & 48.14 & 48.01 & 48.09 & $0.97 \%$ & 1.003 & $0.7 \%$ & 1.000 & $1.2 \%$ \\
\hline May-14 & 48.1 & $0.6 \%$ & 48.14 & 47.92 & 48.04 & $0.95 \%$ & 1.005 & $0.6 \%$ & 0.999 & $1.1 \%$ \\
\hline Jun-14 & 48.1 & $0.6 \%$ & 48.42 & 48.06 & 48.22 & $1.01 \%$ & 1.007 & $0.7 \%$ & 1.002 & $1.2 \%$ \\
\hline Oct-14 & 48.1 & $0.6 \%$ & 48.55 & 48.53 & 48.54 & $0.97 \%$ & 1.000 & $0.5 \%$ & 1.009 & $1.2 \%$ \\
\hline & Average & & $\begin{array}{l}48.25 \\
0.5 \%\end{array}$ & $\begin{array}{l}48.12 \\
0.6 \%\end{array}$ & 48.19 & & 1.003 & & \multicolumn{2}{|l|}{1.002} \\
\hline
\end{tabular}

Table 2: Repeated measurements of the Sb-CRM. Fluences measured by DetA \& DetB for independent calibration procedures between June 2013 and October 2014. "Uncertainties" are the combined standard uncertainties. See text. 


\section{Discussion}

Table 3 gives the position (in channel number) of the peaks ( $\mathrm{Au} \& \mathrm{Ni}$ ) and edges ( $\mathrm{Si} \& \mathrm{O}$ ) observed in the experimental spectra obtained from the $\mathrm{Au} / \mathrm{Ni} / \mathrm{SiO}_{2} / \mathrm{Si}$ calibration sample at two different incident beam energies: namely, $1531.9 \mathrm{keV}$ (Figure 2) and $3080.6 \mathrm{keV}$ (see Fig.1 of the companion work ${ }^{7}$ ). The kinematical factors are given by the RBS formalism ${ }^{1,4}$, in this case for a scattering angle of $173.4^{\circ}$ (DetA). The scattered energies shown in the Table are corrected for the energy losses occurring during the in-path to the scattering event and the out-path towards the detector: that is, for the two energies, about $2.4 \mathrm{keV}(2.0 \mathrm{keV})$ for the Ni signal (due to the Au layer) and about $5.4 \mathrm{keV}(4.8 \mathrm{keV}$ ) for the Si \& O signals (due to the Au \& Ni layers) at $1.5 \mathrm{MeV}(3 \mathrm{MeV})$. The PHD energy losses for this detector are calculated considering a dead layer of 870 TFU of Si and the non-ionising energy loss model given by Lennard ${ }^{20}$.

Considering the scattered energies (that is, neglecting the PHD) for determining the electronic gain and offset one finds:

$$
\begin{array}{lll}
E_{C h}=3.1458 \times C h+68.9 \mathrm{keV} & \text { at } 1.5 \mathrm{MeV} & \text { Eq.4 } \\
E_{C h}=3.1557 \times C h+67.9 \mathrm{keV} & \text { at } 3.0 \mathrm{MeV} & \text { Eq.5 }
\end{array}
$$

The offset is unexpectedly high at about $70 \mathrm{keV}$ where the direct measurement using an electronic pulser indicated $(2.4 \pm 10.8) \mathrm{keV}$ for this detector (DetA). Treating these spectra as energy spectra also causes the apparent gain to be a function of incident beam energy: the variation between the gains determined at $1.5 \mathrm{MeV}$ and $3.0 \mathrm{MeV}$ is about $0.3 \%$.

Considering the detected energies of Table 3 (that is, scattered energies corrected for the PHD) one finds:

$$
\begin{array}{lll}
E_{C h}=3.1831 \times C h-5.4 \mathrm{keV} & \text { at } 1.5 \mathrm{MeV} & \text { Eq.6 } \\
E_{C h}=3.1846 \times C h-3.0 \mathrm{keV} & \text { at } 3.0 \mathrm{MeV} & \text { Eq.7 }
\end{array}
$$

The derived electronic offset is now in close agreement with the direct measurement performed with an electronic pulser. Moreover, the electronic gains determined at $1.5 \mathrm{MeV}$ and $3.0 \mathrm{MeV}$ are indistinguishable (difference lower than $0.05 \%$ ) and in agreement with the electronic gain given by our calibration method (Table 1). This result demonstrates how the PHD can linearise the electronic gain of the pulse-height spectrometry system.

Moreover, the comparison of Eqs. 4 \& 6 (or Eqs. 5 \& 7) highlights the size of the error on the electronic gain determination when handling the data as energy- rather than pulse-height spectra: the electronic gain variation is $1.2 \%(0.9 \%)$ at $1.5 \mathrm{MeV}(3.0 \mathrm{MeV})$ ! Finding a smaller deviation at higher incident beam energy simply reflects the lower PHD correction at higher energies (Figure 3 ). The trouble with gain errors of about $1 \%$ and greater is that the peaks and edges now cannot all be fitted exactly, and the incentive to be careful is reduced, increasing the expected error! Experience shows that it is shockingly easy to get $2 \%$ or even larger errors in the gain determination. 


\begin{tabular}{|c|c|c|c|c|c|c|c|}
\hline $\begin{array}{l}\text { Incident } \\
\text { beam } \\
\text { energy }\end{array}$ & Element & $\begin{array}{c}\text { Kinematical } \\
\text { factor }\end{array}$ & $\begin{array}{c}\text { Scattered } \\
\text { energy } \\
\text { (keV) }\end{array}$ & $\begin{array}{l}\text { Position } \\
\text { (Channel) }\end{array}$ & $\begin{array}{l}\text { Dead } \\
\text { layer }\end{array}$ & $\begin{array}{l}\text { D (keV) } \\
\text { Non-ionising } \\
\text { processes }\end{array}$ & $\begin{array}{c}\text { Detected } \\
\text { energy } \\
\text { (keV) }\end{array}$ \\
\hline \multirow{4}{*}{$1531.9 \mathrm{keV}$} & $\mathrm{Au}$ & 0.922170 & 1412.7 & 428.2 & 47.5 & 10.8 & 1354.4 \\
\hline & $\mathrm{Ni}$ & 0.761624 & 1166.7 & 347.0 & 50.9 & 10.5 & 1102.9 \\
\hline & $\mathrm{Si}$ & 0.564358 & 864.5 & 250.6 & 55.3 & 10.1 & 793.7 \\
\hline & $\mathrm{O}$ & 0.360922 & 552.9 & 152.9 & 58.9 & 9.6 & 479.2 \\
\hline \multirow{4}{*}{$3080.6 \mathrm{keV}$} & $\mathrm{Au}$ & 0.922170 & 2840.8 & 878.8 & 34.2 & 12.0 & 2794.6 \\
\hline & $\mathrm{Ni}$ & 0.761624 & 2344.2 & 720.3 & 37.8 & 11.6 & 2294.8 \\
\hline & $\mathrm{Si}$ & 0.564358 & 1733.9 & 529.8 & 43.5 & 11.1 & 1679.2 \\
\hline & $\mathrm{O}$ & 0.360922 & 1107.0 & 328.4 & 51.7 & 10.5 & 1044.9 \\
\hline
\end{tabular}

Table 3: Effect of the PHD on the electronic gain determination. The kinematical factors are calculated for a scattering angle of $173.4^{\circ}$ (DetA). The positions of the peaks and edges come from the spectra obtained from the $\mathrm{Au} / \mathrm{Ni} / \mathrm{SiO}_{2} / \mathrm{Si}$ calibration sample at $1531.9 \mathrm{keV}$ (Figure 2) and $3080.6 \mathrm{keV}$ (not shown). The scattered energies are corrected for the energy losses occurring in the Au \& Ni layers (see text: thicknesses are respectively 10 \& 20 TFU). The PHD energy losses are calculated for 870 TFU of Si dead layer and the non-ionising model given by Lennard ${ }^{20}$.

Obtaining the PHD correction is therefore of primary importance for correctly calibrating the pulseheight spectrometry system. In our calibration procedure presented above, this is achieved by closely fitting the silicon ( $\left.\mathrm{Si} \& \mathrm{Si} \mid \mathrm{SiO}_{2}\right)$ and oxygen $\left(\mathrm{O} \& \mathrm{Si} \mid \mathrm{SiO}_{2}\right)$ interface signals. Considering Figure 2, the comparison of the oxide/silicon interface in the $\mathrm{Si}$ and $\mathrm{O}$ signals emphasises detector non-linearities, as is very obvious if the energy thickness of the $\mathrm{SiO}_{2}$ layer (in channel number) is compared for the $\mathrm{Si}$ and $\mathrm{O}$ signals: they are in the ratio $51 / 43$ or 1.18. But at this beam energy the energy loss factors for these two signals are only $1 \%$ different! If the spectra are treated as energy spectra (that is, ignoring the PHD) the trailing edge of the $O$ signal cannot be fitted properly, as was already emphasised previously ${ }^{4}$; conversely, this trailing edge signal can be used to determine the PHD, as we do here using a dataset acquired on a wide energy range for probing different parts of the PHD function (Figure 3).

It is worth noting that the energy loss factors are also non-linear with the energy (Figure 6). They are consequently different for the $\mathrm{O}$ and $\mathrm{Si}$ signals, and the evaluation of the PHD relies on the accuracy of the $\mathrm{SiO}_{2}$ (molecular) stopping power measurements. Moreover, Figure 6 shows that significant discrepancies exist between the various databases available in the literature. However, although the variation of the energy loss factor for the $\mathrm{O}$ and $\mathrm{Si}$ signals is large when considering the whole dataset (20-25\% between $250 \mathrm{keV}$ and $1.8 \mathrm{MeV}$, regardless of the database used), this variation is rather small for a given incident beam energy $(\leq 1 \%)$. For that reason, each spectrum is separately fitted in our calibration method, allowing one to compensate any error in the stopping power function by adjusting the $\mathrm{SiO}_{2}$ thickness used in the simulations. The accuracy of the PHD determination in our calibration method is therefore only limited by the eye of the analyst who needs to evaluate the goodness of the fit: that is, about $5 \%$ (evaluated by trial-and-error). We have not yet found a sufficiently sensitive objective test. 


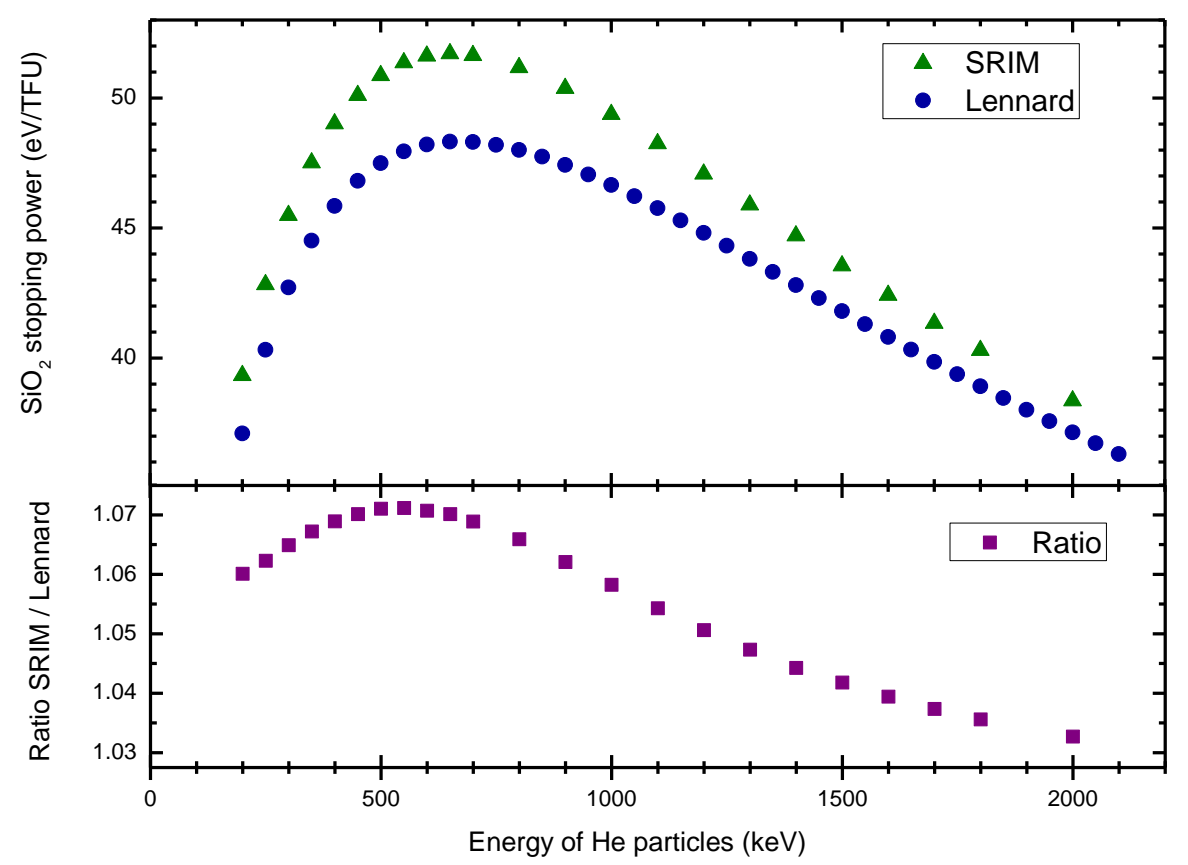

Figure 6: Experimental molecular stopping power of ${ }^{4} \mathrm{He}$ in silica. The upper part shows the values from Lennard et al ${ }^{23}$ compared to SRIM ${ }^{17}$ using Bragg's rule ${ }^{30}$. The lower part shows the ratio of these two measurements.

The procedure described here results in the excellent fits to the spectra shown in Figs. 1 \& 2 . Table 1 summarises the fitted values obtained at the end of the procedure. The whole dataset (36 spectra, 2 detectors) is finally fitted with dead layers fixed at 870 \& 590 TFU of Si for DetA \& DetB respectively, resulting in a mean electronic gain of $(3.1838 \pm 0.0029) \mathrm{keV} / \mathrm{ch}$ for DetA and $(2.9790 \pm 0.0021) \mathrm{keV} / \mathrm{ch}$ for DetB, where the expanded uncertainties are given as the SE99 on the mean (see Eq.3: this is an expanded Type A estimate with a coverage factor $k=3$ in terms of the GUM ${ }^{6}$ ). This is an accuracy far better than $0.1 \%$ for the electronic gain determination of both detectors! It should be noted that similar results could be obtained with a reduced dataset providing that a similar energy range is covered by the dataset: for instance, 9 pairs of spectra acquired between 1.0 and $2.0 \mathrm{MeV}$. The small SD of the detector resolution ( $\sim 3 \%)$, the electronic offset $(<0.4 \mathrm{keV})$ and the $\chi^{2}$ values reported in Table 1 also demonstrate that the whole dataset is consistently fitted when the PHD is properly determined.

In fact, the combined uncertainty of the gain is dominated by the uncertainty of the determination of the PHD. In the companion work we determined the covariance of the PHD and gain by trialand-error, finding that $20 \%$ variation of the PHD gives a gain variation of $0.4 \%$. Then assuming (conservatively) linear behaviour, the sensitivity of $5 \%$ in the PHD for this more precise work (also determined by trial-and-error) implies a gain variation of $0.1 \%$. This is confirmed by a numerical determination of the covariance.

The results obtained in this work have been validated against the Sb-implant CRM: the measured fluence is directly proportional to the electronic gain (Eq.2), but both detectors ought to give the same Sb-fluence since they are measuring the same sample. Table 2 gives the Sb-fluences measured by both detectors on several occasions. The uncertainty associated with the ratio DetA/DetB leaves out all systematic contributions which are identical for both detectors: namely, 
$B_{3-5}$ and $A_{5-6}$ in the Uncertainty Budget described at length elsewhere ${ }^{1,4}$ and given in the Annexe for convenience. Since we are aiming to validate the electronic gain, the uncertainty $A_{3}$ is also disregarded. For each measurement, the ratio DetA/DetB is indistinguishable from unity: that is, the discrepancy from unity is lower than the uncertainty associated with the measurement. Thus the error on the electronic gain (if any) is negligible in comparison to the uncertainty on the ratio. We conclude that the uncertainty on the electronic gain ought to be lower than $0.2 \%$ (i.e. a third of the average uncertainty of the ratio DetA/DetB), in agreement with our estimate (SE99 on the electronic gain mean in Table 1). The agreement between the certified value and the mean fluences measured by DetA and DetB gives further confidence in the electronic gain determination. The mean Average/Certified fluences ratio is found to be $1.002 \pm 0.005$ : indistinguishable from unity.

Finally, it should be noted that the starting point (i.e. iteration \#1 in Figure 5) comes from the GVM calibration procedure described in the companion work ${ }^{7}$. The variation of the dead layer thicknesses between the first and last iteration is found to be about $2.5 \%$ and $14 \%$ for DetA and DetB, respectively. This result is in agreement with the previous conclusions ${ }^{7}$ stating that the PHD cannot be determined at an accuracy much better than $20 \%$ when handling only the dataset of spectra acquired around 3.0 MeV. The very close value found for the DetA dead layer in this work simply reflects that this detector has been used many times (thus the PHD value was already well known), while DetB was a brand new detector. Similarly, the variation of the electronic gains derived from the GVM calibration procedure ${ }^{7}$ or in this work is found to be $0.30 \%$ for the DetA and $0.25 \%$ for the DetB, consistent with the previous claim ${ }^{7}$ (i.e. electronic gain is determined at about $0.4 \%$ handling only the dataset acquired around $3.0 \mathrm{MeV}$ ).

\section{Conclusion}

We have presented a complete method of calibrating the free parameters for Rutherford backscattering spectrometry at the best traceable accuracy for the certification of ion implanted fluences. We have shown that by following the procedure described in this work the PHD per detector can be determined at about $5 \%$, giving $0.1 \%$ as the covariance of the gain of the pulseheight spectrometry system (again, per detector). For a fixed PHD the gain itself is determined very precisely indeed, with a standard uncertainty of only $0.033 \%$. Thus, the standard uncertainty introduced by the gain is $0.11 \%$, dominated by the determination of the PHD.

This estimate of the uncertainty (Type A in terms of GUM ${ }^{6}$, see Table 1) has been shown to be consistent with the repeated analysis of the Sb-implant CRM. The results presented in this work are derived from a dataset of 36 spectra (18 energies, 2 detectors), but we believe that similar results could be achieved with a reduced dataset covering a similar energy range (for instance, 9 pairs spectra acquired between 1.0 and $2.0 \mathrm{MeV}$ ) for properly probing the PHD function (Figure 2). The dead layer thickness of each detector can be derived at better than $5 \%$, which is of primary importance for properly determine the electronic gain: neglecting the PHD in the data processing leads to errors of $1 \%$ or larger in the electronic gain! 
This calibration method is relatively fast, requiring only about half an hour for the measurement of the ADC offset, about 90 mins for the acquisition of the datasets around $3 \& 1.5 \mathrm{MeV}$, and less than half a day for the data processing. It is suitable for any kind of accelerator system, either Tandem or single ended; stabilised either by slit feedback at the analysing magnet or by feedback directly from the generating voltmeter on the high voltage terminal. 


\section{Annexe}

\begin{tabular}{|c|c|c|c|c|c|}
\hline & & Type of Error & $\begin{array}{c}\text { Cornell } \\
\text { detector } \\
\mathbf{A}\end{array}$ & $\begin{array}{c}\text { IBM } \\
\text { detector } \\
\text { B } \\
\end{array}$ & Comment \\
\hline & Pileup correction & \multirow{4}{*}{$\begin{array}{c}\text { Non- } \\
\text { Systematic }\end{array}$} & $0.4 \%$ & $0.7 \%$ & \\
\hline $\mathrm{B}_{1}$ & Uncertainty of pileup correction & & $0.04 \%$ & $0.07 \%$ & $10 \%$ from shape fitting accuracy \\
\hline $\mathrm{A}_{1}$ & Counting statistics, implant signal & & $0.22 \%$ & $0.15 \%$ & Whole dataset \\
\hline $\mathrm{A}_{2}$ & Counting statistics, a-Si signal & & $0.14 \%$ & $0.10 \%$ & Whole dataset \\
\hline $\mathrm{B}_{2}$ & Scattering angle & \multirow{2}{*}{ Systematic } & $0.07 \%$ & $0.40 \%$ & $0.2^{\circ}$ and $\sim 1 / \sin ^{4}(\theta / 2)$ and $1 / \cos (\theta)$ \\
\hline $\mathrm{A}_{3}$ & Electronic calibration uncertainty & & $0.09 \%$ & $0.07 \%$ & From the calibration on $\mathrm{Au} / \mathrm{Ni} / \mathrm{SiO} 2 / \mathrm{Si}$ sample \\
\hline $\mathrm{U}_{1}$ & Relative uncertainty & & $0.3 \%$ & $0.4 \%$ & \\
\hline $\mathrm{U}_{2}$ & $\begin{array}{l}\text { Relative uncertainty of average of two } \\
\text { detectors }\end{array}$ & & \multicolumn{2}{|c|}{$0.2 \%$} & Relative accuracy \\
\hline $\mathrm{B}_{3}$ & Beam energy & \multirow{5}{*}{ Systematic } & \multicolumn{2}{|c|}{$0.20 \%$} & $\begin{array}{l}\text { From Anal. Methods 6, 2014, 120-129 } \\
\text { http://dx.doi.org/10.1039/c3ay41398e }\end{array}$ \\
\hline $\mathrm{A}_{4}$ & Disagreement between both detectors & & \multicolumn{2}{|c|}{$0.13 \%$} & $\begin{array}{l}\text { From Anal. Methods 6, 2014, 120-129 } \\
\text { http://dx.doi.org/10.1039/c3ay41398e }\end{array}$ \\
\hline $\mathrm{B}_{4}$ & Pileup uncertainty (from model) & & \multicolumn{2}{|c|}{$0.20 \%$} & From Anal. Chem. 84 (2012) 6061-6069 \\
\hline $\mathrm{B}_{5}$ & Code Uncertainty & & \multicolumn{2}{|c|}{$0.20 \%$} & From IAEA Intercomparison \\
\hline $\mathrm{B}_{6}$ & Rutherford cross-section & & \multicolumn{2}{|c|}{$0.27 \%$} & $\begin{array}{l}\text { Screening correction for } \mathrm{Sb} \\
\text { (same for both detectors) }\end{array}$ \\
\hline $\mathrm{U}_{3}$ & Combined extra Type B uncertainty & & \multicolumn{2}{|c|}{$0.5 \%$} & \\
\hline $\mathrm{A}_{5}$ & \multirow{2}{*}{ Si stopping power } & \multirow{2}{*}{ Systematic } & \multirow{2}{*}{\multicolumn{2}{|c|}{$\begin{array}{l}0.06 \% \\
0.80 \%\end{array}$}} & Influence of the $\mathrm{S}_{\mathrm{Si}}$ uncertainty on the $\mathrm{Sb}$ counts \\
\hline $\mathrm{A}_{6}$ & & & & & Influence of the $\mathrm{S}_{\mathrm{Si}}$ uncertainty on the a-Si yield \\
\hline $\mathrm{U}_{4}$ & $\begin{array}{c}\text { Total combined standard uncertainty } \\
\text { (dataset) }\end{array}$ & & \multicolumn{2}{|c|}{$1.0 \%$} & Absolute accuracy \\
\hline
\end{tabular}

Typical Uncertainty Budget obtained for the Sb-implant measurement using a $1.5 \mathrm{MeV}^{4} \mathrm{He}$ beam $(\mathrm{Q}=100 \mu \mathrm{C})$. Further details about the different components can be found elsewhere ${ }^{1,4}$. 


\section{References}

1. J. L. Colaux and C. Jeynes, High accuracy traceable Rutherford backscattering spectrometry of ion implanted samples, Anal. Methods, 2014, 6, 120-129.

2. J. L. Colaux, C. Jeynes, K. C. Heasman and R. M. Gwilliam, Certified ion implantation fluence by high accuracy RBS, Analyst, 2015, http://dx.doi.org/10.1039/C4AN02316A

3. C. Jeynes, M. J. Bailey, N. J. Bright, M. E. Christopher, G. W. Grime, B. N. Jones, V. V. Palitsin and R. P. Webb, "Total IBA" - Where are we?, Nucl Instrum Meth B, 2012, 271, 107-118.

4. C. Jeynes, N. P. Barradas and E. Szilágyi, Accurate Determination of Quantity of Material in Thin Films by Rutherford Backscattering Spectrometry, Anal Chem, 2012, 84, 6061-6069.

5. K. H. Ecker, U. Wätjen, A. Berger, L. Persson, W. Pritzkow, M. Radtke and H. Riesemeier, RBS, SY$X R F$, INAA and ICP-IDMS of antimony implanted in silicon - A multi-method approach to characterize and certify a reference material, Nucl Instrum Meth B, 2002, 188, 120-125.

6. Guide to the Expression of Uncertainty in Measurement (GUM), International Organization for Standardization, Geneva, Switzerland, 1995.

7. J. L. Colaux, G. Terwagne and C. Jeynes, On the traceably accurate voltage calibration of electrostatic accelerators, Nucl Instrum Meth B, 2015, http://dx.doi.org/10.1016/j.nimb.2015.02.048.

8. A. F. Gurbich and C. Jeynes, Evaluation of non-Rutherford proton elastic scattering cross-section for magnesium, Nucl Instrum Meth B, 2007, 265, 447-452.

9. F. Munnik, A. J. M. Plompen, J. Räisänen and U. Wätjen, Stopping powers of 200-3000 keV ${ }^{4} \mathrm{He}$ and 550-1750 keV ${ }^{1} \mathrm{H}$ ions in Vyns, Nucl Instrum Meth B, 1996, 119, 445-451.

10. A. Simon, C. Jeynes, R. P. Webb, R. Finnis, Z. Tabatabian, P. J. Sellin, M. B. H. Breese, D. F. Fellows, R. van den Broek and R. M. Gwilliam, The new Surrey ion beam analysis facility, Nucl Instrum Meth B, 2004, 219, 405-409.

11. J. P. Zhang, R. J. Wilson, P. L. F. Hemment, A. Claverie, F. Cristiano, P. Salles, J. Q. Wen, J. H. Evans, A. R. Peaker and G. J. Parker, Regrowth Behavior of $S_{1} i_{1-X} G e_{x} /$ Si Structures Formed by Ge+ IonImplantation and Post Amorphization, Nucl Instrum Meth B, 1994, 84, 222-228.

12. G. Lulli, E. Albertazzi, M. Bianconi, R. Nipoti, M. Cervera, A. Camera and C. Cellini, Stopping and damage parameters for Monte Carlo simulation of MeV implants in crystalline Si, J Appl Phys, 1997, 82, 5958-5964.

13. N. P. Barradas, C. Jeynes and R. P. Webb, Simulated annealing analysis of Rutherford backscattering data, Appl Phys Lett, 1997, 71, 291-293.

14. N. P. Barradas and C. Jeynes, Advanced physics and algorithms in the IBA DataFurnace, Nucl Instrum Meth B, 2008, 266, 1875-1879.

15. H. H. Andersen, F. Besenbacher, P. Loftager and W. Moller, Large-Angle Scattering of Light-Ions in the Weakly Screened Rutherford Region, Phys Rev A, 1980, 21, 1891-1901.

16. J. F. Ziegler, Srim-2003, Nucl Instrum Meth B, 2004, 219, 1027-1036.

17. J. F. Ziegler, M. D. Ziegler and J. P. Biersack, SRIM - The stopping and range of ions in matter (2010), Nucl Instrum Meth B, 2010, 268, 1818-1823.

18. S. L. Molodtsov and A. F. Gurbich, Simulation of the pulse pile-up effect on the pulse-height spectrum, Nucl Instrum Meth B, 2009, 267, 3484-3487.

19. C. Pascual-Izarra and N. P. Barradas, Introducing routine pulse height defect corrections in IBA, Nucl Instrum Meth B, 2008, 266, 1866-1870.

20. W. N. Lennard, S. Y. Tong, G. R. Massoumi and L. Wong, On the Calibration of Low-Energy Ion Accelerators, Nucl Instrum Meth B, 1990, 45, 281-284.

21. N. P. Barradas, C. Jeynes and S. M. Jackson, RBS/simulated annealing analysis of buried SiCO $_{x}$ layers formed by implantation of O into cubic silicon carbide, Nucl Instrum Meth B, 1998, 136, 1168-1171.

22. C. Pascual-Izarra, M. Bianconi, G. Lulli and C. Summonte, Stopping power of SiO2 for 0.2-3.0 MeV He ions, Nucl Instrum Meth B, 2002, 196, 209-214.

23. W. N. Lennard, H. Xia and J. K. Kim, Revisiting the stopping powers of Si and SiO2 for He-4 ions: 0.5-2.0 MeV, Nucl Instrum Meth B, 2004, 215, 297-307.

24. G. Konac, S. Kalbitzer, C. Klatt, D. Niemann and R. Stoll, Energy loss and straggling of $H$ and He ions of keV energies in Si and C, Nucl Instrum Meth B, 1998, 136, 159-165.

25. C. Jeynes, N. P. Barradas, M. J. Blewett and R. P. Webb, Improved ion beam analysis facilities at the University of Surrey, Nucl Instrum Meth B, 1998, 136, 1229-1234. 
26. International Union of Pure and Applied Chemistry (IUPAC) - Compendium of Chemical Terminology, 2nd ed. (the "Gold Book"). Compiled by A.D. McNaught and A. Wilkinson. Blackwell Scientific Publications, Oxford., 1997.

27. W. Hosler and R. Darji, ON THE NONLINEARITY OF SILICON DETECTORS AND THE ENERGY CALIBRATION IN RBS, Nucl Instrum Meth B, 1994, 85, 602-606.

28. Z. Siketić, I. B. Radović, E. Alves and N. P. Barradas, Stopping power of ${ }^{11} \mathrm{~B}$ in Si and $\mathrm{TiO}_{2} \mathrm{measured}$ with a bulk sample method and Bayesian inference data analysis, Nucl Instrum Meth B, 2010, 268, 1768-1771.

29. C. Jeynes and A. C. Kimber, High-Accuracy Data from Rutherford Backscattering SpectraMeasurements of the Range and Straggling of 60-400 Kev as Implants into Si, J Phys D Appl Phys, 1985, 18, L93-L97.

30. W. H. Bragg and M. A. Elder, On the alpha particles of radium, and their loss of range in passing through various atoms and molecules, Philos Mag, 1905, 10, 318-340. 